\title{
Geochemistry of Rare Earth Elements in Sediment of Central India
}

\author{
Bharat Lal Sahu', Keshaw Prakash Rajhans' ${ }^{1}$, Shobhana Ramteke1, \\ Khageshwar Singh Patel ${ }^{1 *}$, Irena Wysocka ${ }^{2}$, Irena Jaron ${ }^{2}$ \\ ${ }^{1}$ School of Studies in Chemistry/Environmental Science, Pt. Ravishankar Shukla University, Raipur, India \\ ${ }^{2}$ Polish Geological Institute, Warsaw, Poland \\ Email: patelks_55@hotmail.com
}

Received 30 January 2016; accepted 15 April 2016; published 18 April 2016

Copyright (C) 2016 by authors and Scientific Research Publishing Inc.

This work is licensed under the Creative Commons Attribution International License (CC BY).

http://creativecommons.org/licenses/by/4.0/

\section{(c) (i) Open Access}

\begin{abstract}
The rare earth elements (REE) are of great interests due to wide industrial applications. In the present work, geochemistry of REE in the contaminated pond sediment of Ambagarh Chowki, central India is described. The concentration ( $\mathrm{n}=24$ ) of $\mathrm{La}, \mathrm{Ce}, \mathrm{Pr}, \mathrm{Nd}, \mathrm{Eu}, \mathrm{Sm}, \mathrm{Gd}, \mathrm{Tb}, \mathrm{Dy}, \mathrm{Ho}, \mathrm{Er}, \mathrm{Tm}$, $\mathrm{Yb}, \mathrm{Lu}, \mathrm{Th}$ and $\mathrm{U}$ was ranged from 16 - 119, 41 - 163, 4.1 - 28, 16 - 99, 0.7 - 2.7, 3.2 - 18, 2.7 - 16, 0.4 2.3, 2.2 - 13, $0.4-2.4,1.1-6.1,0.2-0.8,0.9-4.8,0.13-0.70,6-25$ and $0.46-1.86 \mathrm{mg} / \mathrm{kg}$ with mean value of $42 \pm 9,89 \pm 13,9.4 \pm 1.9,34 \pm 7,1.2 \pm 0.2,6.3 \pm 1.2,5.4 \pm 1.0,0.8 \pm 0.1,4.2 \pm 0.8,0.8 \pm 0.2$, $2.0 \pm 0.4,0.3 \pm 0.1,1.6 \pm 0.3,0.24 \pm 0.04,11 \pm 2$ and $1.14 \pm 0.13 \mathrm{mg} / \mathrm{kg}$, respectively. The concentration ratio of $\sum$ LREE/ $\Sigma$ HREE, $\mathrm{La} / \mathrm{Y}$ and $\mathrm{Th} / \mathrm{U}$ are discussed. The spatial distribution and sources of the REE are described.
\end{abstract}

\section{Keywords}

Rare Earth Element, Sediment, Geochemistry, Sources

\section{Introduction}

The rare earth elements (REE) including actinide have wide industrial applications in several fields i.e. nuclear energy as fuel and modifier, organic synthesis as catalyst, ceramics as coloring agents, X-ray tubes as phosphors, etc. [1] [2]. The distribution of REE in various environmental materials i.e. soil and sediments was reported [3]-[13]. Recently, the health hazards of the REE were highlighted [14] [15]. The environment of the Ambagarh Chowki block, central India is seriously contaminated with As and heavy metals [16]. In this work, the geochemistry of the REE in pond sediments of Ambagarh Chowki block, Rajnandgaon district, central India is described.

\footnotetext{
${ }^{*}$ Corresponding author.
}

How to cite this paper: Sahu, B.L., Rajhans, K.P., Ramteke, S., Patel, K.S., Wysocka, I. and Jaron, I. (2016) Geochemistry of Rare Earth Elements in Sediment of Central India. Journal of Environmental Protection, 7, $705-714$. 


\section{Materials and Methods}

\subsection{Study Area}

Arsenic and other heavy metals are deposited in the environment of Ambagarh Chowki block, Rajnandgaon district, Chhattisgarh, India over area $\approx 3000 \mathrm{~km}^{-2}$. The Ambagarh Chowki block falls in a tribal belt with population of $\approx 0.1$ million. This block covers 155 villages and towns which suffering with serious health hazards due to contamination of the environment with toxic elements. In this work, 24 ponds lie in different 11 villages (locations) of Ambagarh Chowki block were selected for the investigation of the REE contamination (Figure 1).

\subsection{Sampling}

The composite surface sediment sample $(0-10 \mathrm{~cm})$ was collected from five points in the equal ratio from each pond as prescribed in literature [17]. For the depth profile studies, the samples at the depth profile of 0 - 10, 10 20 and $20-30 \mathrm{~cm}$ of the Bhadsena pond was collected.

\subsection{Analysis}

The samples were dried, crushed and sieved out the particles of size $\leq 1 \mathrm{~mm}$. A $0.25 \mathrm{~g}$ of each sediment sample was digested with $8 \mathrm{ml}$ aqua regia in the closed microwave vessel for $15 \mathrm{~min}$. An aliquot of the extract was diluted with deionized water for the further analysis. The Thermo Fisher ICP-OES and ICP-MS (Polish Geological Institute, NRI, Central Chemical Laboratory, and Warsaw) were employed for analysis of the elements. The NCS DC 73382 CRM sediment sample was used for the quality control. The background levels of the REE reported in the upper earth crust were used for computation of the enrichment factor $\left(E_{f}\right)$ by using following equation [18].

$$
\mathrm{E}_{\mathrm{f}}=\left\{\left[\mathrm{X}_{\mathrm{s}}\right] /\left[\mathrm{Al}_{\mathrm{s}}\right]\right\} /\left\{\left[\mathrm{X}_{\mathrm{e}}\right] /\left[\mathrm{Al}_{\mathrm{e}}\right]\right\}
$$

where, $\mathrm{X}_{\mathrm{s}}, \mathrm{Al}_{\mathrm{s}}, \mathrm{X}_{\mathrm{e}}$ and $\mathrm{Al}_{\mathrm{e}}$ are concentrations of metal and $\mathrm{Al}$ in the soil and earth crust, respectively.

\section{Results and Discussion}

The sediment was found to be colored from light brown ( $\mathrm{LBr}$ ) to dark brown ( $\mathrm{DBr}$ ), Table 1 . The $\mathrm{pH}$ value of the extract was ranged from $6.5-8.1$ with mean value of $7.2 \pm 0.2$.

\subsection{Concentration of Elements}

The concentrations of 49 elements in 24 pond sediments are shown in Tables 1-4. The concentration of elements i.e. $\mathrm{Fe}, \mathrm{Al}, \mathrm{Ca}, \mathrm{Mg}, \mathrm{K}, \mathrm{Mn}, \mathrm{Ti}, \mathrm{Na}, \mathrm{P}, \mathrm{S}, \mathrm{Ba}, \mathrm{V}, \mathrm{Zn}, \mathrm{Cu}$, As, Ni, Cr, Co, Sr, Pb, Rb, Ga, Li, Be, Mo, Ag, Cd, Sn, Sb, Cs, Bi, Th, U, Sc, Y, La, Ce, Pr, Nd, Eu, Sm, Gd, Tb, Dy, Ho, Er, Tm, Tb and Lu was ranged from 25,400 65,900, 11,800 - 34,700, 1500 - 16,100, 1500 - 30,200, 1200 - 4500, 256 - 1660, 62 - 735, 90 - 830, 90 - 570, 50 790, 88 - 304, 29 - 166, 32 - 100, 28 - 89, 10 - 53, 19 - 340, 24 - 1298, 9 - 76, 10 - 69, 10 - 40, 12 - 46, 7 - 15, 5 18, 0.9 - 3.4,0.14 - 1.32, 0.07 - 2.36, 0.05 - 0.39, 0.8 - 2.6, 0.27 - 0.97, 0.5 - 2.2, 0.10 - 0.30, 6.1 - 25, 0.5 - 1.9, 3.0 - 25, 11 - 61, 16 - 119, 41 - 163, 4.0 - 28, 16 - 99, 0.7 - 2.7, 3.2 - 18, $2.7-16,0.4-2.3,2.2-13,0.4-2.4,1.1$ - 6.1, $0.15-0.83,0.9-4.8$ and $0.13-0.70 \mathrm{mg} / \mathrm{kg}$ with mean value of $44842 \pm 5133,23813 \pm 2819,5133 \pm 1418$, $6850 \pm 2581,2708 \pm 243,759 \pm 158,254 \pm 79,355 \pm 93,268 \pm 45,285 \pm 81,196 \pm 21,84 \pm 16,63 \pm 7,52 \pm 6$, $22 \pm 5,75 \pm 30,177 \pm 118,30 \pm 7,26 \pm 6,23 \pm 3,26 \pm 3,11 \pm 1,12 \pm 2,1.6 \pm 0.2,0.63 \pm 0.14,0.67 \pm 0.23$, $0.13 \pm 0.03,1.5 \pm 0.2,0.44 \pm 0.05,1.2 \pm 0.2,0.15 \pm 0.03,11 \pm 2,1.1 \pm 0.1,11 \pm 2,20 \pm 4,42 \pm 8,42 \pm 8,89 \pm$ $13,9 \pm 2,34 \pm 7,1.2 \pm 0.2,6.3 \pm 1.2,5.4 \pm 1.0,0.77 \pm 0.15,4.2 \pm 0.8,0.8 \pm 0.2,2.0 \pm 0.4,0.27 \pm 0.05,1.6 \pm 0.3$ and $0.24 \pm 0.04 \mathrm{mg} / \mathrm{kg}$, respectively. Among them, the highest concentration of Fe was marked. They occurred in following decreasing order in the sediment: $\mathrm{Fe}<\mathrm{Al}<\mathrm{Mg}<\mathrm{Ca}<\mathrm{K}<\mathrm{Mn}<\mathrm{Na}<\mathrm{S}<\mathrm{P}<\mathrm{Ti}<\mathrm{Ba}<\mathrm{Ce}<\mathrm{Cr}$ $<\mathrm{V}<\mathrm{Ni}<\mathrm{Zn}<\mathrm{Cu}<\mathrm{La}<<\mathrm{Nd}<\mathrm{Co}<\mathrm{Sr} \approx \mathrm{Rb}<\mathrm{Pb}<\mathrm{As}<\mathrm{Y}<\mathrm{Li}<\mathrm{Ga} \approx \mathrm{Th} \approx \mathrm{Sc}<\mathrm{Pr}<\mathrm{Sm}<\mathrm{Gd}<\mathrm{Dy}<\mathrm{Er}$ $<\mathrm{Yb} \approx \mathrm{Be}<\mathrm{Sn}<\mathrm{Eu} \approx \mathrm{Cs}<\mathrm{U}<\mathrm{Tb} \approx \mathrm{Ho}<\mathrm{Ag}<\mathrm{Mo}<\mathrm{Sb}<\mathrm{Tm}<\mathrm{Lu}<\mathrm{Cd} \approx \mathrm{Bi}$.

\subsection{Concentration of REE}

The concentration of the LREE (i.e. La, Ce, Pr, Nd, Eu and Sm) and HREE (i.e. Gd, Tb, Dy, Ho, Er, Tm, Yb 
Table 1. Concentration of major element in sediments, mg/kg.

\begin{tabular}{|c|c|c|c|c|c|c|c|c|c|c|}
\hline S. No. & Pond location & Color & $\mathrm{Fe}$ & $\mathrm{Al}$ & $\mathrm{Ca}$ & $\mathrm{Mg}$ & $\mathrm{K}$ & Mn & $\mathrm{Ti}$ & $\mathrm{Na}$ \\
\hline 1 & Murethitola-1 & $\mathrm{LBr}$ & 62,700 & 31,400 & 5800 & 6300 & 2900 & 778 & 735 & 160 \\
\hline 2 & Murethitola-2 & $\mathrm{LBr}$ & 64,900 & 34,700 & 7100 & 6800 & 3000 & 956 & 586 & 540 \\
\hline 3 & Murethitola-3 & $\mathrm{RBr}$ & 32,700 & 17,200 & 1800 & 1700 & 2100 & 447 & 166 & 90 \\
\hline 4 & Netamtola-1 & DB & 54,700 & 32,200 & 9400 & 14,300 & 2500 & 892 & 394 & 630 \\
\hline 5 & Netamtola-2 & B & 48,000 & 32,200 & 12,500 & 9200 & 4000 & 1474 & 140 & 830 \\
\hline 6 & Netamtola-3 & LB & 53,800 & 30,000 & 16,100 & 9300 & 1300 & 959 & 455 & 740 \\
\hline 7 & Kaudikasa-1 & $\mathrm{DBr}$ & 49,800 & 30,600 & 7600 & 10,800 & 4000 & 1109 & 264 & 490 \\
\hline 8 & Kaudikasa-2 & $\mathrm{RBr}$ & 49,500 & 19,200 & 2000 & 2800 & 4500 & 467 & 254 & 180 \\
\hline 9 & Kaudikasa-3 & LB & 25,400 & 19,200 & 4700 & 4800 & 2400 & 256 & 64 & 220 \\
\hline 10 & Ghorda-1 & $\mathrm{Br}$ & 45,600 & 29,400 & 3700 & 7700 & 2900 & 1660 & 173 & 130 \\
\hline 11 & Ghorda-2 & LB & 41,000 & 28,600 & 5800 & 30,200 & 1600 & 1148 & 556 & 100 \\
\hline 12 & Ghorda-3 & $\mathrm{LBr}$ & 57,700 & 34,300 & 7600 & 17,900 & 1200 & 671 & 571 & 150 \\
\hline 13 & Metepar-1 & $\mathrm{Br}$ & 43,600 & 26,700 & 4800 & 6600 & 2000 & 856 & 105 & 110 \\
\hline 14 & Metepar-2 & $\mathrm{LBr}$ & 59,000 & 23,400 & 3400 & 4900 & 2200 & 878 & 313 & 730 \\
\hline 15 & Metepar-3 & $\mathrm{LBr}$ & 65,900 & 25,100 & 3600 & 5000 & 2900 & 540 & 320 & 270 \\
\hline 16 & Bhadsena & $\mathrm{LBr}$ & 38,000 & 21,500 & 2100 & 2500 & 3000 & 350 & 68 & 340 \\
\hline 17 & Sonsaitola-1 & LB & 28,000 & 18,600 & 4800 & 3700 & 3200 & 694 & 62 & 280 \\
\hline 18 & Sonsaitola-2 & $\mathrm{LBr}$ & 28,000 & 14,100 & 2500 & 1800 & 3400 & 388 & 92 & 330 \\
\hline 19 & Sonsaitola-3 & $\mathrm{DBr}$ & 32,500 & 14,800 & 2500 & 2200 & 3800 & 435 & 98 & 600 \\
\hline 20 & Meregaon & $\mathrm{Br}$ & 50,000 & 19,600 & 3100 & 2500 & 2200 & 1393 & 185 & 250 \\
\hline 21 & Joratarai & $\mathrm{DBr}$ & 53,100 & 23,600 & 3300 & 5100 & 2700 & 853 & 133 & 360 \\
\hline 22 & Thailitola-1 & $\mathrm{LBr}$ & 26,900 & 11,800 & 2500 & 2600 & 2500 & 285 & 125 & 230 \\
\hline 23 & Thailitola-2 & $\mathrm{RBr}$ & 33,500 & 14,700 & 1500 & 1500 & 1700 & 369 & 163 & 140 \\
\hline 24 & Jadutola & LB & 31,900 & 18,600 & 5000 & 4200 & 3000 & 350 & 73 & 630 \\
\hline
\end{tabular}

Table 2. Chemical characteristics of metal in pond sediment, $\mathrm{mg} / \mathrm{kg}$.

\begin{tabular}{|c|c|c|c|c|c|c|c|c|c|c|c|c|}
\hline S. No. & $\mathrm{P}$ & $\mathrm{S}$ & $\mathrm{Ba}$ & V & $\mathrm{Zn}$ & $\mathrm{Cu}$ & As & $\mathrm{Ni}$ & $\mathrm{Cr}$ & Co & $\mathrm{Sr}$ & $\mathrm{Pb}$ \\
\hline 1 & 300 & 80 & 217 & 144 & 76 & 64 & 40 & 51 & 45 & 30 & 29 & 17 \\
\hline 2 & 380 & 380 & 259 & 160 & 88 & 71 & 17 & 58 & 64 & 44 & 39 & 24 \\
\hline 3 & 160 & 70 & 129 & 59 & 42 & 53 & 33 & 29 & 39 & 13 & 12 & 20 \\
\hline 4 & 430 & 790 & 179 & 86 & 90 & 89 & 16 & 133 & 301 & 41 & 42 & 24 \\
\hline 5 & 420 & 540 & 304 & 87 & 80 & 71 & 12 & 85 & 136 & 39 & 54 & 22 \\
\hline 6 & 170 & 70 & 150 & 166 & 53 & 49 & 12 & 43 & 56 & 33 & 69 & 12 \\
\hline 7 & 340 & 600 & 264 & 96 & 88 & 73 & 20 & 108 & 232 & 42 & 30 & 27 \\
\hline 8 & 320 & 320 & 212 & 48 & 100 & 48 & 53 & 30 & 61 & 18 & 16 & 40 \\
\hline 9 & 220 & 400 & 179 & 50 & 50 & 41 & 15 & 55 & 120 & 18 & 21 & 23 \\
\hline
\end{tabular}




\section{Continued}

\begin{tabular}{|c|c|c|c|c|c|c|c|c|c|c|c|c|}
\hline 10 & 160 & 360 & 300 & 79 & 52 & 47 & 12 & 196 & 635 & 76 & 17 & 39 \\
\hline 11 & 570 & 90 & 218 & 67 & 54 & 36 & 10 & 340 & 1298 & 62 & 26 & 22 \\
\hline 12 & 330 & 110 & 161 & 93 & 65 & 63 & 15 & 194 & 658 & 30 & 28 & 10 \\
\hline 13 & 200 & 70 & 222 & 95 & 53 & 46 & 19 & 51 & 51 & 28 & 35 & 19 \\
\hline 14 & 170 & 190 & 204 & 142 & 60 & 56 & 17 & 48 & 45 & 38 & 27 & 15 \\
\hline 15 & 180 & 180 & 211 & 152 & 68 & 56 & 18 & 49 & 45 & 26 & 27 & 13 \\
\hline 16 & 330 & 310 & 188 & 55 & 65 & 37 & 12 & 31 & 33 & 13 & 13 & 32 \\
\hline 17 & 220 & 300 & 213 & 50 & 40 & 35 & 28 & 38 & 50 & 20 & 25 & 19 \\
\hline 18 & 290 & 470 & 140 & 38 & 54 & 37 & 28 & 23 & 29 & 12 & 12 & 22 \\
\hline 19 & 220 & 370 & 154 & 47 & 44 & 53 & 30 & 28 & 30 & 14 & 14 & 17 \\
\hline 20 & 170 & 50 & 221 & 77 & 45 & 42 & 11 & 37 & 47 & 34 & 18 & 27 \\
\hline 21 & 340 & 300 & 209 & 98 & 87 & 59 & 25 & 77 & 133 & 36 & 22 & 31 \\
\hline 22 & 150 & 180 & 118 & 29 & 48 & 28 & 34 & 22 & 24 & 11 & 10 & 23 \\
\hline 23 & 90 & 60 & 88 & 48 & 32 & 32 & 45 & 19 & 30 & 9 & 10 & 24 \\
\hline 24 & 280 & 550 & 165 & 52 & 67 & 50 & 11 & 49 & 82 & 25 & 21 & 37 \\
\hline
\end{tabular}

Table 3. Chemical characteristics of minor element in pond sediment, $\mathrm{mg} / \mathrm{kg}$.

\begin{tabular}{|c|c|c|c|c|c|c|c|c|c|c|c|c|c|}
\hline S. No. & $\mathrm{Rb}$ & $\mathrm{Ga}$ & $\mathrm{Li}$ & $\mathrm{Be}$ & Mo & $\mathrm{Ag}$ & $\mathrm{Cd}$ & Sn & $\mathrm{Sb}$ & Cs & $\mathrm{Bi}$ & Th & $\mathrm{U}$ \\
\hline 1 & 32 & 14 & 14 & 1.57 & 0.67 & 0.15 & 0.12 & 1.4 & 0.46 & 2.18 & 0.3 & 13.2 & 1.64 \\
\hline 2 & 23 & 15 & 13 & 1.35 & 0.40 & 0.69 & 0.26 & 1.4 & 0.35 & 1.41 & 0.1 & 7.2 & 0.77 \\
\hline 3 & 27 & 8 & 8 & 1.47 & 0.85 & 0.13 & 0.05 & 1.4 & 0.43 & 0.98 & 0.3 & 15.7 & 1.36 \\
\hline 4 & 12 & 11 & 17 & 1.16 & 0.27 & 0.73 & 0.39 & 1.2 & 0.51 & 0.52 & 0.1 & 6.1 & 0.65 \\
\hline 5 & 22 & 12 & 14 & 1.98 & 0.40 & 0.92 & 0.19 & 2.1 & 0.4 & 0.95 & 0.1 & 10.2 & 1.36 \\
\hline 6 & 15 & 10 & 11 & 1.06 & 0.24 & 0.07 & 0.05 & 1.1 & 0.42 & 1.00 & 0.1 & 6.5 & 1.01 \\
\hline 7 & 29 & 12 & 17 & 1.55 & 0.52 & 1.60 & 0.21 & 1.7 & 0.52 & 1.23 & 0.1 & 8.0 & 1.14 \\
\hline 8 & 46 & 11 & 9 & 1.68 & 1.32 & 0.16 & 0.11 & 1.3 & 0.59 & 1.63 & 0.2 & 9.3 & 1.12 \\
\hline 9 & 20 & 7 & 9 & 1.29 & 0.26 & 1.70 & 0.16 & 1.5 & 0.27 & 0.97 & 0.1 & 10.1 & 1.04 \\
\hline 10 & 39 & 13 & 18 & 2.55 & 0.92 & 0.56 & 0.14 & 2.6 & 0.50 & 2.09 & 0.1 & 14.1 & 1.42 \\
\hline 11 & 15 & 9 & 18 & 0.86 & 0.18 & 0.26 & 0.06 & 1.0 & 0.35 & 1.19 & 0.1 & 7.6 & 0.74 \\
\hline 12 & 14 & 12 & 15 & 1.39 & 0.14 & 0.19 & 0.05 & 2.0 & 0.3 & 1.08 & 0.1 & 8.0 & 0.46 \\
\hline 13 & 28 & 10 & 14 & 1.47 & 0.20 & 0.13 & 0.06 & 1.3 & 0.34 & 1.69 & 0.1 & 10.1 & 1.34 \\
\hline 14 & 21 & 12 & 11 & 1.1 & 0.68 & 0.78 & 0.13 & 1.1 & 0.38 & 1.08 & 0.1 & 7.3 & 1.15 \\
\hline 15 & 31 & 13 & 12 & 1.14 & 0.81 & 0.69 & 0.10 & 1.3 & 0.39 & 1.32 & 0.2 & 8.7 & 1.01 \\
\hline 16 & 44 & 12 & 12 & 3.37 & 0.84 & 0.6 & 0.10 & 2.6 & 0.43 & 1.46 & 0.2 & 24.9 & 1.86 \\
\hline 17 & 25 & 8 & 9 & 1.58 & 0.39 & 0.19 & 0.06 & 1.4 & 0.34 & 1.1 & 0.1 & 11.5 & 1.08 \\
\hline 18 & 28 & 9 & 7 & 2.07 & 0.84 & 1.07 & 0.14 & 1.8 & 0.45 & 0.8 & 0.2 & 14.4 & 1.17 \\
\hline 19 & 31 & 9 & 7 & 2.08 & 0.95 & 0.79 & 0.10 & 1.9 & 0.45 & 1.05 & 0.3 & 13.3 & 1.17 \\
\hline 20 & 22 & 10 & 9 & 1.32 & 0.96 & 0.52 & 0.06 & 1.3 & 0.36 & 1.02 & 0.1 & 10.4 & 0.95 \\
\hline 21 & 27 & 11 & 14 & 1.33 & 1.19 & 1.06 & 0.19 & 1.5 & 0.97 & 1.26 & 0.2 & 7.9 & 1.2 \\
\hline 22 & 23 & 7 & 5 & 1.28 & 0.63 & 0.75 & 0.08 & 0.8 & 0.42 & 0.83 & $<0.1$ & 16.9 & 1.33 \\
\hline 23 & 24 & 8 & 7 & 1.61 & 1.04 & 0.07 & 0.05 & 1.0 & 0.49 & 1.08 & 0.1 & 15.1 & 0.84 \\
\hline 24 & 29 & 9 & 9 & 2.24 & 0.53 & 2.36 & 0.19 & 1.9 & 0.38 & 0.91 & 0.1 & 13.2 & 1.64 \\
\hline
\end{tabular}


Table 4. Concentration of lanthanide in pond sediment, $\mathrm{mg} / \mathrm{kg}$.

\begin{tabular}{|c|c|c|c|c|c|c|c|c|c|c|c|c|c|c|c|c|}
\hline S. No. & Sc & $\mathrm{Y}$ & $\mathrm{La}$ & Ce & $\operatorname{Pr}$ & $\mathrm{Nd}$ & $\mathrm{Eu}$ & $\mathrm{Sm}$ & $\mathrm{Gd}$ & $\mathrm{Tb}$ & Dy & Но & Er & $\mathrm{Tm}$ & $\mathrm{Yb}$ & $\mathrm{Lu}$ \\
\hline 1 & 18.0 & 21.0 & 40.0 & 66.0 & 8.7 & 32.0 & 1.3 & 5.9 & 5.2 & 0.8 & 4.1 & 0.8 & 2.1 & 0.3 & 1.6 & 0.2 \\
\hline 2 & 25.0 & 21.0 & 32.0 & 73.0 & 7.3 & 27.0 & 1.3 & 5.3 & 4.7 & 0.7 & 4.1 & 0.8 & 2.2 & 0.3 & 1.8 & 0.3 \\
\hline 3 & 8.0 & 23.0 & 56.0 & 99.0 & 12.9 & 47.0 & 1.6 & 8.6 & 7.0 & 0.9 & 4.9 & 0.9 & 2.2 & 0.3 & 1.5 & 0.2 \\
\hline 4 & 18.0 & 13.0 & 21.0 & 49.0 & 4.8 & 18.0 & 0.8 & 3.4 & 3.1 & 0.4 & 2.6 & 0.5 & 1.4 & 0.2 & 1.2 & 0.2 \\
\hline 5 & 12.0 & 22.0 & 37.0 & 93.0 & 8.3 & 30.0 & 1.1 & 5.8 & 5.0 & 0.8 & 4.4 & 0.8 & 2.2 & 0.3 & 1.8 & 0.3 \\
\hline 6 & 16.0 & 13.0 & 16.0 & 43.0 & 4.1 & 16.0 & 0.8 & 3.3 & 2.9 & 0.5 & 2.6 & 0.5 & 1.4 & 0.2 & 1.2 & 0.2 \\
\hline 7 & 14.0 & 18.0 & 33.0 & 77.0 & 7.5 & 27.0 & 1.1 & 5.2 & 4.5 & 0.7 & 3.6 & 0.7 & 1.8 & 0.2 & 1.4 & 0.2 \\
\hline 8 & 10.0 & 22.0 & 47.0 & 94.0 & 10.8 & 40.0 & 1.5 & 7.2 & 6.1 & 0.9 & 4.5 & 0.8 & 2.0 & 0.3 & 1.4 & 0.2 \\
\hline 9 & 7.0 & 15.0 & 29.0 & 74.0 & 6.3 & 22.0 & 0.8 & 4.2 & 3.7 & 0.6 & 3.1 & 0.6 & 1.6 & 0.2 & 1.3 & 0.2 \\
\hline 10 & 11.0 & 23.0 & 50.0 & 157.0 & 10.5 & 37.0 & 1.2 & 7.0 & 6.1 & 0.9 & 4.7 & 0.9 & 2.4 & 0.3 & 1.8 & 0.3 \\
\hline 11 & 8.0 & 11.0 & 22.0 & 64.0 & 4.6 & 17.0 & 0.7 & 3.2 & 2.7 & 0.4 & 2.2 & 0.4 & 1.1 & 0.2 & 0.9 & 0.1 \\
\hline 12 & 23.0 & 13.0 & 31.0 & 41.0 & 6.4 & 22.0 & 0.8 & 3.9 & 3.2 & 0.5 & 2.6 & 0.5 & 1.3 & 0.2 & 1.1 & 0.2 \\
\hline 13 & 11.0 & 18.0 & 28.0 & 84.0 & 7.1 & 26.0 & 1.0 & 5.2 & 4.4 & 0.7 & 3.7 & 0.7 & 1.8 & 0.3 & 1.5 & 0.2 \\
\hline 14 & 15.0 & 16.0 & 23.0 & 55.0 & 5.7 & 22.0 & 1.0 & 4.2 & 3.9 & 0.6 & 3.4 & 0.6 & 1.7 & 0.2 & 1.3 & 0.2 \\
\hline 15 & 18.0 & 20.0 & 28.0 & 53.0 & 6.9 & 26.0 & 1.2 & 5.1 & 4.7 & 0.7 & 3.9 & 0.8 & 2.0 & 0.3 & 1.5 & 0.2 \\
\hline 16 & 9.0 & 61.0 & 119.0 & 163.0 & 28.3 & 99.0 & 2.7 & 18.0 & 16.0 & 2.3 & 13.0 & 2.4 & 6.1 & 0.8 & 4.8 & 0.7 \\
\hline 17 & 7.0 & 21.0 & 40.0 & 91.0 & 9.0 & 33.0 & 1.1 & 6.3 & 5.5 & 0.8 & 4.3 & 0.8 & 2.1 & 0.3 & 1.7 & 0.2 \\
\hline 18 & 5.0 & 28.0 & 65.0 & 126.0 & 14.2 & 51.0 & 1.4 & 9.2 & 7.9 & 1.1 & 6.0 & 1.1 & 2.8 & 0.4 & 2.2 & 0.3 \\
\hline 19 & 6.0 & 24.0 & 54.0 & 111.0 & 11.9 & 43.0 & 1.3 & 7.9 & 6.6 & 0.9 & 5.0 & 0.9 & 2.4 & 0.3 & 1.9 & 0.3 \\
\hline 20 & 9.0 & 18.0 & 45.0 & 108.0 & 9.8 & 36.0 & 1.3 & 6.4 & 5.4 & 0.7 & 3.8 & 0.7 & 1.7 & 0.2 & 1.2 & 0.2 \\
\hline 21 & 12.0 & 18.0 & 32.0 & 73.0 & 7.5 & 28.0 & 1.2 & 5.5 & 4.9 & 0.7 & 3.7 & 0.7 & 1.8 & 0.2 & 1.4 & 0.2 \\
\hline 22 & 3.0 & 15.0 & 59.0 & 110.0 & 11.7 & 41.0 & 0.9 & 6.9 & 5.4 & 0.7 & 3.3 & 0.6 & 1.5 & 0.2 & 1.1 & 0.2 \\
\hline 23 & 5.0 & 14.0 & 53.0 & 99.0 & 10.8 & 38.0 & 1.0 & 6.7 & 5.2 & 0.6 & 3.1 & 0.5 & 1.4 & 0.2 & 1.1 & 0.2 \\
\hline 24 & 5.0 & 20.0 & 56.0 & 131.0 & 11.1 & 39.0 & 1.1 & 6.9 & 5.8 & 0.8 & 4.2 & 0.8 & 1.9 & 0.3 & 1.5 & 0.2 \\
\hline
\end{tabular}

and Lu) was ranged from 82 - 431 and 8 - $46 \mathrm{mg} / \mathrm{kg}$ with mean value of $182 \pm 30$ and $15 \pm 3 \mathrm{mg} / \mathrm{kg}$, respectively. The ratio of LREE/HREE and Lu/Y was found to be in the range of 9 - 18 and 14 - 56 with mean value of $12 \pm 1$ and $27 \pm 4$, respectively. The concentration of REE and LREE/HREE and Lu/Y ratio were found to be higher than values reported in river sediments of the other regions of the World [3]-[11].

The concentration of $\mathrm{Th}$ and $\mathrm{U}$ in the sediments $(\mathrm{n}=24)$ was ranged from $6.1-25$ and $0.46-1.86 \mathrm{mg} / \mathrm{kg}$ with mean value of $11 \pm 2$ and $1.14 \pm 0.13 \mathrm{mg} / \mathrm{kg}$, respectively. The Th/U ratio was ranged from 6 - 28 with mean value of $11 \pm 2$. The concentration of actinide elements in the studied area was found to be comparable to values reported in soil of other regions of the country [12] [13] (Figure 2).

\subsection{Spatial Variations and Sources}

The sediment samples were collected from 24 ponds which situated in 11 locations (i.e. villages) of Ambagarh Chowki block. The content of the REE in the pond sediments of 11 locations are shown in Figure 3. The highest content of the REE in the pond sediment of Bhadsena was observed. The REE content in sediment was found to increase as the depth profile was increased from 0 - $30 \mathrm{~cm}$, may be due to geogenic origin and less binding with organic components of the sediment, Figure 4. 


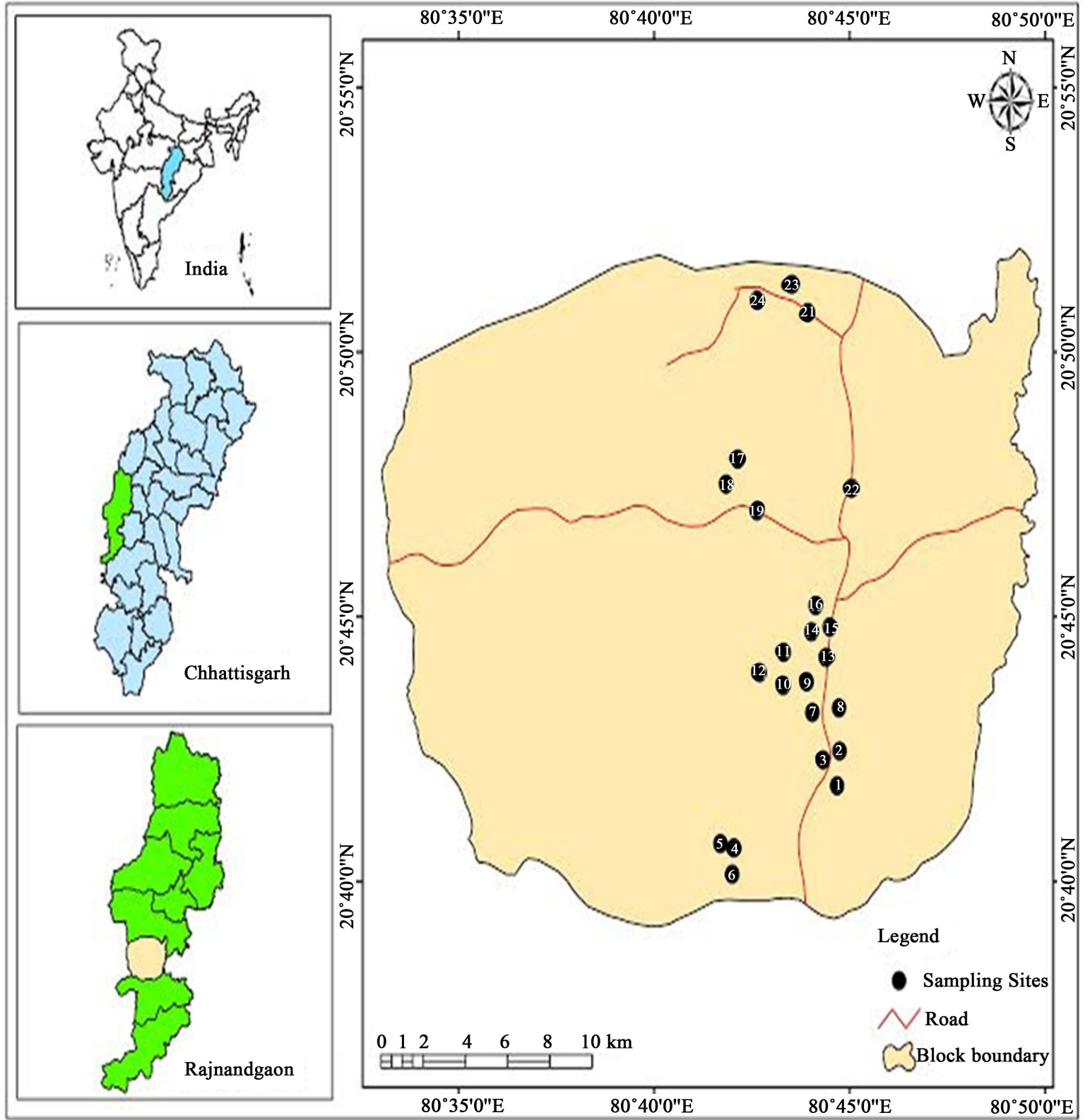

Figure 1. Representation of sampling locations (i.e. Murethitola-1, Murethitola-2, Murethitola- 3, Netamtola- 1, Netamtola-2, Netamtola-3, Kaudikasa-1, Kaudikasa-2, Kaudikasa-3, Ghorda-1, Ghorda-2, Ghorda-3, Metepar-1, Metepar-2, Metepar-3, Bhadsena, Sonsaitola-1, Sonsaitola-2, Sonsaitola-3, Meregaon, Joratarai,Thailitola-1,Thailitola-2 and Jadutola) in Ambagarh Chowki.

The correlation matrix of the REE is summarized in Table 5. All REE inclusive of Eu, Th and U are correlated well in the sediment, indicating origin from the similar sources likely to geogenic origin. They were partially correlated with elements i.e. Be, K, Rb, Cs, Mo, As, Sb, Bi, Sn, and Ag. Their negative correlations with elements i.e. Na, Mg, Ca, Ba, Sr, Al, Ga, V, Cr, Fe, Mn, Co, Ni, Cu, Zn, Cd and Pb were observed.

\subsection{Enrichment}

The $\mathrm{E}_{\mathrm{f}}$ value for Sc, Y, La, Ce, Pr, Eu, Nd, Sm, Gd, Tb, Dy, Ho, Er, Tm, Yb, Lu, Th and U in the sediment was ranged from 0.8 - 4.2, 1.5 - 9.4,1.7 - 13, 1.4 - 12, 1.7 - 13, 1.6 - 9,1.7 - 12, 1.6 - 12, 1.9 - 13, 1.6 - 11,1.6 - 11, 1.6 - 9, $1.4-9,1.4-9,1.4-8,1.1-7,1.1-11$ and $0.4-3$ with mean value of $2.5 \pm 0.4,4 \pm 1,6 \pm 1,5 \pm 1,5 \pm 1,4$ 
- La $\square$ Ce $\quad \operatorname{Pr} \square \mathrm{Nd}$

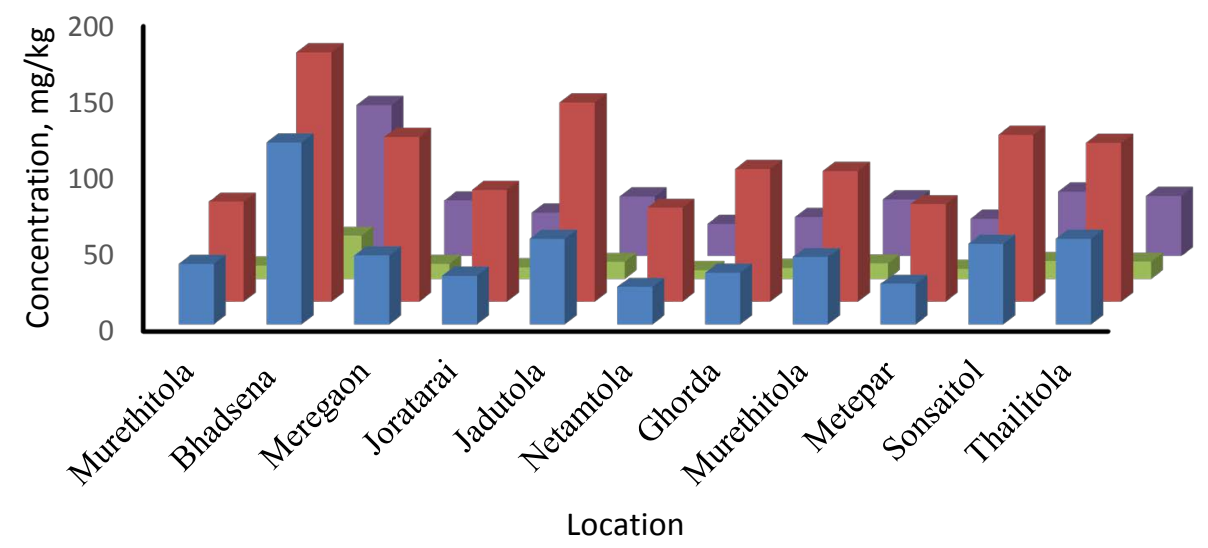

(a)

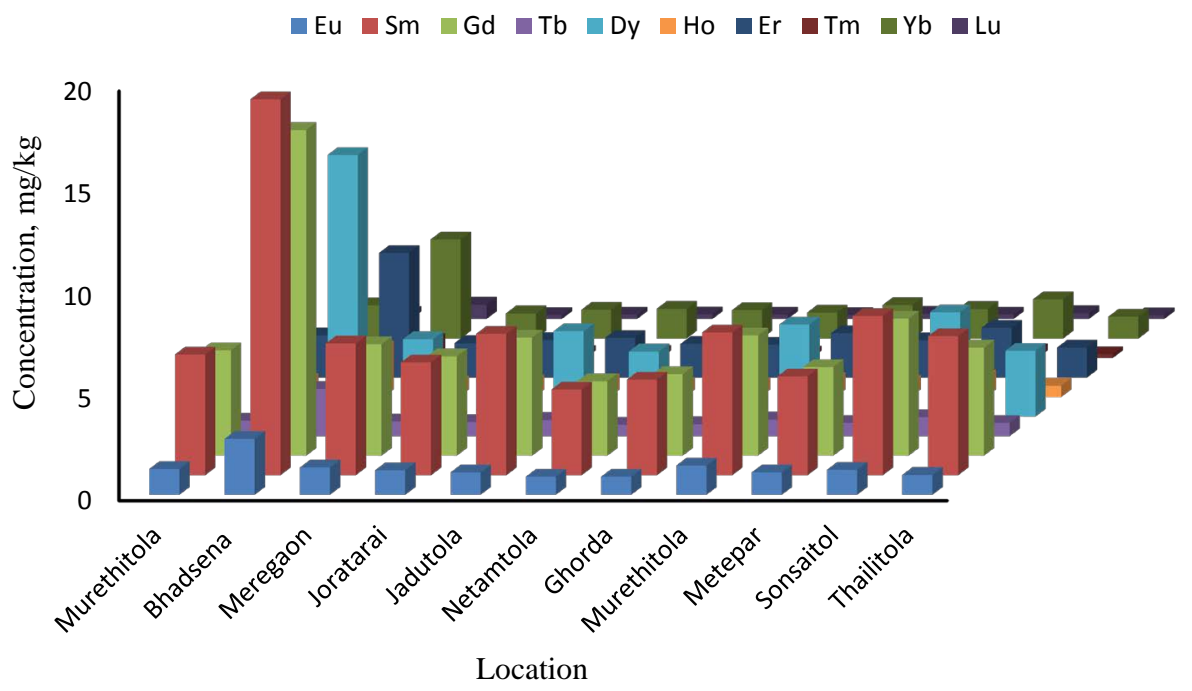

(b)

Figure 2. Spatial variation of REE concentration in sediment of Ambagarh Chowki.

Th

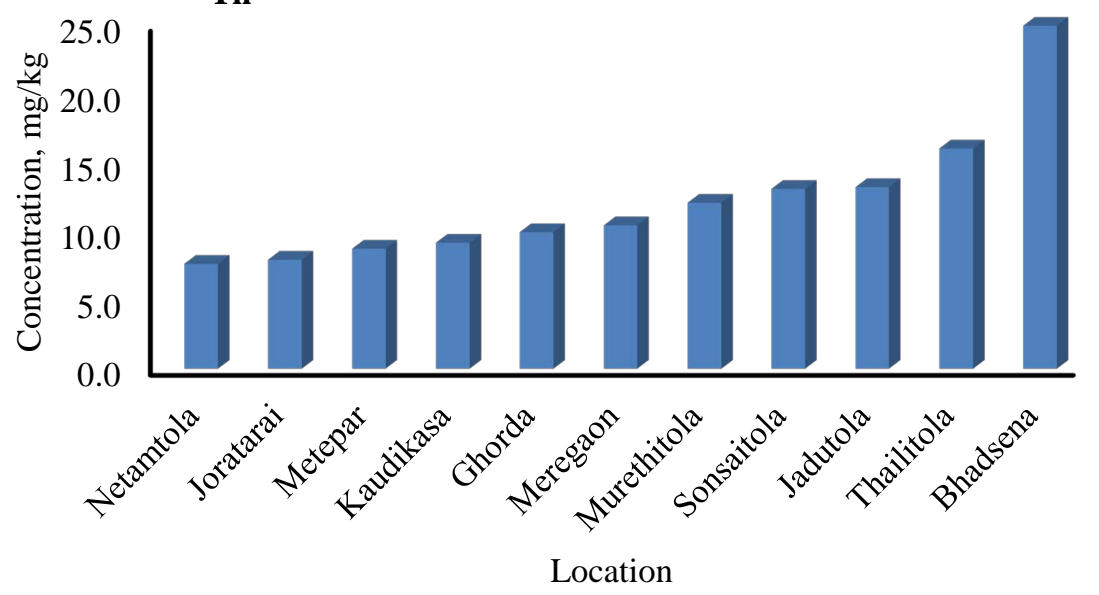

(a) 


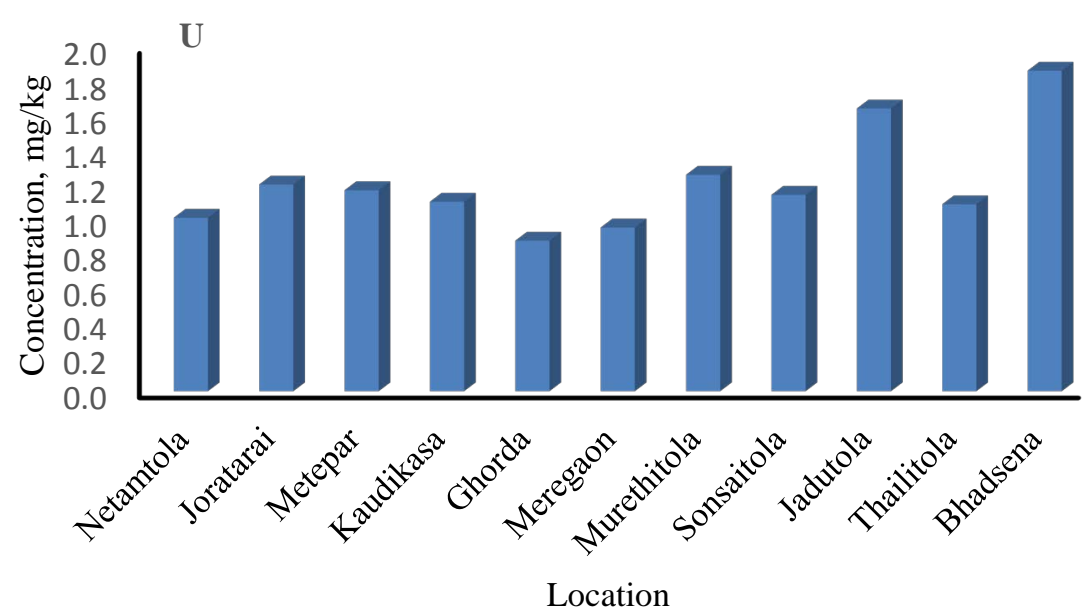

(b)

Figure 3. Spatial variation in concentration of actinide in sediment.

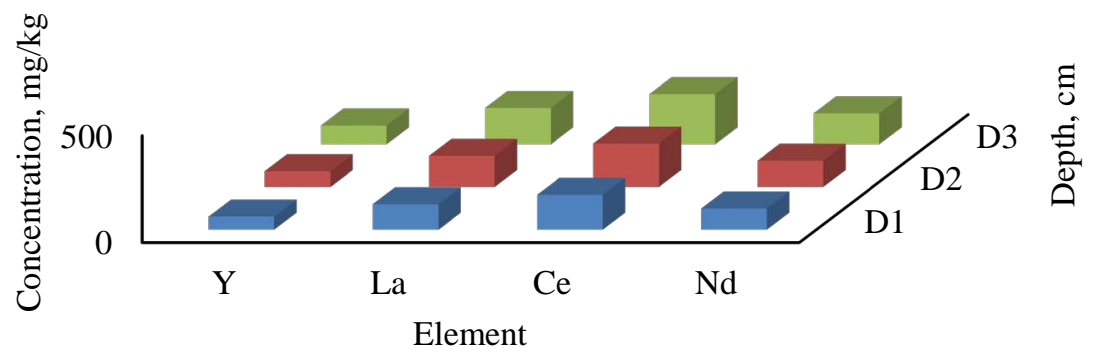

D1 D2 D3

(a)

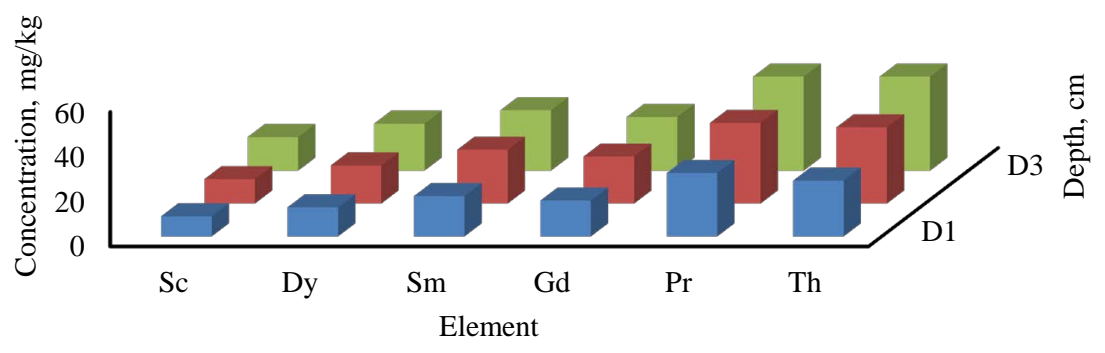

$\square \mathrm{D} 1 \backsim \mathrm{D} 2 \square \mathrm{D} 3$

(b)

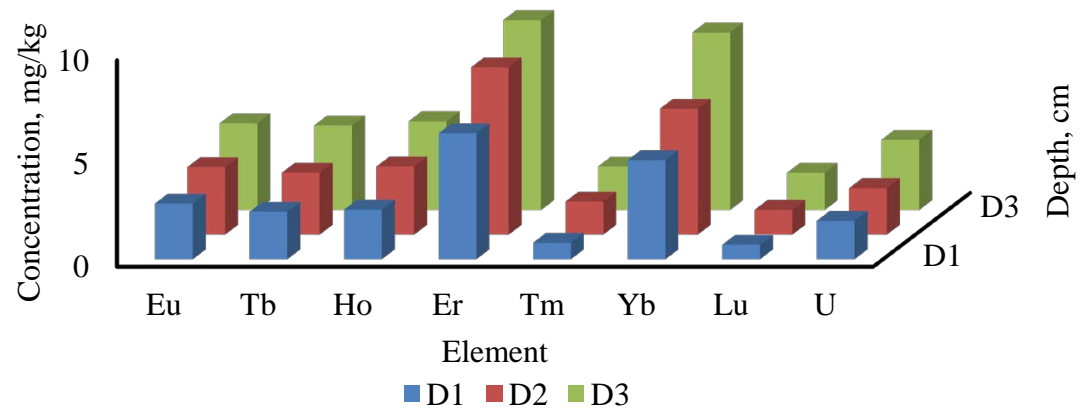

(c)

Figure 4. Depth profile studies of elements in Bhadsena pond sediment. D1 = $0-10 \mathrm{~cm}, \mathrm{D} 2=10-20 \mathrm{~cm}, \mathrm{D} 3=20-30 \mathrm{~cm}$. 
Table 5. Correlation matrix of REE.

\begin{tabular}{|c|c|c|c|c|c|c|c|c|c|c|c|c|c|c|}
\hline & $\mathrm{La}$ & $\mathrm{Ce}$ & $\operatorname{Pr}$ & $\mathrm{Nd}$ & $\mathrm{Eu}$ & $\mathrm{Sm}$ & $\mathrm{Gd}$ & $\mathrm{Tb}$ & Dy & Но & $\mathrm{Er}$ & $\mathrm{Tm}$ & $\mathrm{Yb}$ & $\mathrm{Lu}$ \\
\hline $\mathrm{La}$ & 1.00 & & & & & & & & & & & & & \\
\hline Ce & 0.84 & 1.00 & & & & & & & & & & & & \\
\hline $\operatorname{Pr}$ & 0.99 & 0.80 & 1.00 & & & & & & & & & & & \\
\hline $\mathrm{Nd}$ & 0.99 & 0.80 & 1.00 & 1.00 & & & & & & & & & & \\
\hline $\mathrm{Eu}$ & 0.84 & 0.63 & 0.90 & 0.91 & 1.00 & & & & & & & & & \\
\hline $\mathrm{Sm}$ & 0.97 & 0.78 & 0.99 & 1.00 & 0.93 & 1.00 & & & & & & & & \\
\hline Gd & 0.95 & 0.76 & 0.98 & 0.99 & 0.94 & 1.00 & 1.00 & & & & & & & \\
\hline $\mathrm{Tb}$ & 0.92 & 0.72 & 0.96 & 0.96 & 0.95 & 0.98 & 0.99 & 1.00 & & & & & & \\
\hline Dy & 0.89 & 0.69 & 0.94 & 0.94 & 0.94 & 0.96 & 0.98 & 1.00 & 1.00 & & & & & \\
\hline Но & 0.87 & 0.67 & 0.92 & 0.93 & 0.94 & 0.95 & 0.97 & 0.99 & 1.00 & 1.00 & & & & \\
\hline Er & 0.85 & 0.65 & 0.91 & 0.91 & 0.93 & 0.94 & 0.96 & 0.99 & 1.00 & 1.00 & 1.00 & & & \\
\hline $\mathrm{Tm}$ & 0.83 & 0.64 & 0.89 & 0.89 & 0.90 & 0.92 & 0.94 & 0.98 & 0.99 & 0.99 & 1.00 & 1.00 & & \\
\hline $\mathrm{Yb}$ & 0.81 & 0.61 & 0.87 & 0.87 & 0.89 & 0.91 & 0.93 & 0.97 & 0.98 & 0.99 & 0.99 & 1.00 & 1.00 & \\
\hline $\mathrm{Lu}$ & 0.83 & 0.63 & 0.89 & 0.89 & 0.90 & 0.92 & 0.94 & 0.97 & 0.98 & 0.99 & 1.00 & 1.00 & 1.00 & 1.00 \\
\hline
\end{tabular}

$\pm 1,5 \pm 1,5 \pm 1,5 \pm 1,4 \pm 1,4 \pm 1,4 \pm 1,3 \pm 1,3 \pm 1,3 \pm 1,3 \pm 1,4 \pm 1$ and $1.7 \pm 0.3$, respectively. They were moderately enriched in the sediment. Their highest enrichments were marked in sediment of the Bhadsena village.

\section{Conclusion}

The REE were moderately enriched in all ponds and their enrichments were found to decrease as the mass number of the REE was increased. The concentration of all REE in the sediment was increased vertically, may be due to origin from the rock weathering. Their highest contents were marked in the sediment of Bhadsena village, Rajnandgaon, India.

\section{Acknowledgements}

We are thankful to the Alexander von Humboldt Foundation, Bonn for financial support to one of author: KSP. Our sincere thanks are also extended to the UGC, New Delhi for awarding Rajiv Gandhi Scholarship to students: BLS, KPR and SR.

\section{References}

[1] Cotton, S. (2006) Lanthanide and Actinide Chemistry. Wiley, Hoboken. http://dx.doi.org/10.1002/0470010088

[2] Ramesh, R., Ramanathan, Al., Ramesh, S., Purvaja, R. and Subramanian, V. (2000) Distribution of Rare Earth Elements and Heavy Metals in the Surficial Sediments of the Himalayan River System. Geochemical Journal, 34, $295-319$. http://dx.doi.org/10.2343/geochemj.34.295

[3] Bounouira, H., Choukri, A., Elmoursli, R.C., Hakam, O.K. and Chakiri, S. (2007) Distribution of the Rare Earth Elements in the Sediments of the Bouregreg River (Morocco) Using the Instrumental Neutron Activation Analysis (INAA). Journal of Applied Sciences and Environmental Management, 11, 57-60.

[4] Piper, D.Z. and Bau, M. (2013) Normalized Rare Earth Elements in Water, Sediments, and Wine: Identifying Sources and Environmental Redox Conditions. American Journal of Analytical Chemistry, 4, 69-83. http://dx.doi.org/10.4236/ajac.2013.410A1009

[5] Antonina, A.N., Shazili, N.A.M., Kamaruzzaman, B.Y., Ong, M.C., Rosnan, Y. and Sharifah, F.N. (2013) Geochemistry of the Rare Earth Elements (REE) Distribution in Terengganu Coastal Waters: A Study Case from Redang Island Marine Sediment. Open Journal of Marine Science, 3, 154-159. http://dx.doi.org/10.4236/ojms.2013.33017 
[6] Obaje, S.O., Akpoborie, I.A., Ugbe, F.C. and Onugba, A. (2015) Rare Earth and Trace Elements Distribution in Sediments of River Gora, Minna Area, North-Central Nigeria: Implication for Provenance. Earth Science Research, 4, 103112. http://dx.doi.org/10.5539/esr.v4n1p103

[7] Araújo, M.F., Corredeira, C. and Gouveia, A. (2007) Distribution of the Rare Earth Elements in Sediments of the Northwestern Iberian Continental Shelf. Journal of Radioanalytical and Nuclear Chemistry, 271, 255-260. http://dx.doi.org/10.1007/s10967-007-0201-6

[8] Xu, Y.Y., Song, J.M., Duan, L.Q., Li, X.G., Zhang, Y. and Sun, P.Y. (2010) Environmental Geochemistry Reflected by Rare Earth Elements in Bohai Bay (North China) Core Sediments. Journal of Environmental Monitoring, 12, 15471555. http://dx.doi.org/10.1039/c0em00013b

[9] He, J., Lü, C.W., Xue, H.X., Liang, Y., Bai, S., Sun, Y., Shen, L.L., Mi, N. and Fan, Q.Y. (2010) Species and Distribution of Rare Earth Elements in the Baotou Section of the Yellow River in China. Environmental Geochemistry and Health, 32, 45-58. http://dx.doi.org/10.1007/s10653-009-9264-3

[10] Lee, M., Bae, W., Chung, J., Jung, H.S. and Shim, H. (2008) Seasonal and Spatial Characteristics of Seawater and Sediment at Youngil Bay, Southeast Coast of Korea. Marine Pollution Bulletin, 57, 325-334. http://dx.doi.org/10.1016/j.marpolbul.2008.04.038

[11] Tranchida, G., Oliveri, E., Angelone, M., Bellanca, A., Censi, P., D’Elia, M., Neri, R., Placenti, F., Sprovieri, M. and Mazzola, S. (2011) Distribution of Rare Earth Elements in Marine Sediments from the Strait of Sicily (western Mediterranean Sea): Evidence of Phosphogypsum Waste Contamination. Marine Pollution Bulletin, 62, 182-191.

[12] Maharana, M., Krishnan, N. and Sengupta, D. (2010) Spatial Distribution of Gamma Radiation Levels in Surface Soils from Jaduguda Uranium Mineralization Zone, Jharkhand. Journal of Medical Physics, 35, 235-241. http://dx.doi.org/10.4103/0971-6203.71762

[13] Manigandan, P.K. and Natrajan, K.K. (2014) Activity Concentrations of Natural Radionuclides in Soils of Rainforest Sites in Western Ghats. International Journal of Students Research in Technology and Management, 2, 103-108.

[14] Pagano, G., Guida, M., Tommasi, F. and Oral, R. (2015) Health Effects and Toxicity Mechanisms of Rare Earth Elements-Knowledge Gaps and Research Prospects. Ecotoxicological and Environmental Safety, 115, 40-48. http://dx.doi.org/10.1016/j.ecoenv.2015.01.030

[15] Pagano, G., Aliberti, F., Guida, M., Oral, R., Siciliano A., Trifuoggi, M. and Tommasi, F. (2015) Rare Earth Elements in Human and Animal Health: State of Art and Research Priorities. Environmental Research, 142, 215-220. http://dx.doi.org/10.1016/j.envres.2015.06.039

[16] Patel, K.S., Shrivas, K., Brandt, R., Jakubowski, N., Corns, W. and Hoffmann, P. (2005) Arsenic Contamination in Water, Soil, Sediment and Rice of Central India. Environmental Geochemistry Health, 27, 131-145. http://dx.doi.org/10.1007/s10653-005-0120-9

[17] IAEA-TECDOC 1360 (2003) Collection and Preparation of Bottom Sediment Samples for Analysis of Radionuclides and Trace Elements. 34, 1-130. http://www-pub.iaea.org/MTCD/publications/PDF/te 1360 web.pdf

[18] Rudnick, R.L. and Gao, S. (2003) Vol. 3: The Crust, 3.01-The Composition of the Continental Crust. In: Holland, H.D. and Turekian, K.K., Eds., Treatise on Geochemistry, Elsevier-Pergamon, Oxford, 1-64. http://dx.doi.org/10.1016/b0-08-043751-6/03016-4 